\title{
Examination of the differences between sulforaphane and sulforaphene in colon cancer: A study based on next-generation sequencing
}

\author{
LEI GAO $^{1,2^{*}}$, FENGYING DU ${ }^{3 *}$, JINSHEN WANG ${ }^{3}$, YUHUA ZHAO ${ }^{1,2}$, JUNHUA LIU ${ }^{1,2}$, \\ $\mathrm{DACAI}^{1,2}$, XIAO ZHANG ${ }^{1,2}$, YUTAO WANG ${ }^{1,2}$ and SHUQIU ZHANG $^{1,2}$ \\ ${ }^{1}$ Institute of Quality Standard and Testing Technology for Agro-Products, \\ Shandong Academy of Agricultural Sciences; ${ }^{2}$ Shandong Provincial Key Laboratory of Test Technology on \\ Food Quality and Safety, Jinan, Shandong 250100; ${ }^{3}$ Department of Gastrointestinal Surgery, Shandong Provincial Hospital, \\ Cheeloo College of Medicine, Shandong University, Jinan, Shandong 250021, P.R. China
}

Received July 22, 2020; Accepted June 8, 2021

DOI: $10.3892 / \mathrm{ol} .2021 .12951$

\begin{abstract}
Sulforaphane and sulforaphene are isothiocyanate compounds derived from cruciferous vegetables that have demonstrated antiproliferative properties against colon cancer. However, the underlying mechanism of action of these two compounds has yet to be elucidated. The aim of the present study was to examine the effects of sulforaphane and sulforaphene on colon cancer using next-generation sequencing (NGS). The SW480 colon cancer cell line was cultured with $25 \mu \mathrm{mol} / 1$ sulforaphane or sulforaphene. Total RNA was extracted from the cells following $48 \mathrm{~h}$ of incubation with these compounds, and NGS was performed. Pearson's correlation and principal component analyses were performed on the NGS data in order to determine sample homogeneity followed by hierarchical clustering, chromosomal location, Gene Ontology (GO) and Kyoto Encyclopedia of Genes and Genomes (KEGG) pathway enrichment analyses. A total of 873 probes in the sulforaphene group were differentially expressed compared with the control group. Similarly, 959 probes in the sulforaphane group were differentially expressed compared with the control group. The differentially expressed genes were dispersed on the chromosomes, across 22 pairs of autosomes, as well as the $\mathrm{X}$ and $\mathrm{Y}$ chromosomes. GO and KEGG analyses demonstrated that both drugs affected the 'p53 signaling pathway', 'MAPK signaling
\end{abstract}

Correspondence to: Dr Yutao Wang or Dr Shuqiu Zhang, Institute of Quality Standard and Testing Technology for Agro-Products, Shandong Academy of Agricultural Sciences, 202 Gongyebei Road, Jinan, Shandong 250100, P.R. China

E-mail: 76810875@qq.com

E-mail: zxszsq@163.com

*Contributed equally

Key words: sulforaphane, sulforaphene, colon cancer, next-generation sequencing, Wnt pathway, estrogen pathway pathway', 'FOXO signaling pathway' and 'estrogen signaling pathway', while 'Wnt signaling pathway' was enriched in the sulforaphane group, and 'ubiquitin mediated proteolysis' and 'estrogen signaling pathway' in the sulforaphene group. Thus, sulforaphane and sulforaphene exhibited similar biological activities on colon cancer cells. Sulforaphane and sulforaphene may be associated with Wnt and estrogen signaling, respectively.

\section{Introduction}

Sulforaphane is an isothiocyanate compound derived from cruciferous vegetables. Previous studies have shown that sulforaphane exhibits inhibitory effects on the progression of promyelocytic leukemia, skin, bladder, prostate, colon, pancreatic, liver, lung, nasopharyngeal, ovarian, breast and cervical cancer (1-12). Sulforaphane may inhibit the proliferation and malignant transformation of cancer cells by producing reactive oxygen species, inhibiting cytochrome P450 3A4 and phase-I metabolism enzymes, inhibiting $\mathrm{G}_{1}$ to $\mathrm{S}$-phase progression and $\mathrm{G}_{2} / \mathrm{M}$ phase arrest, as well as activating the intrinsic and extrinsic apoptotic pathways (13-17). Moreover, sulforaphane can achieve efficient glutathione depletion to improve the accumulation of cisplatin in cancer cells (18).

Sulforaphene exhibits a similar structure to sulforaphane with the exception of one carbon-carbon double bond. Both molecules are isothiocyanates derived from cruciferous vegetables (Table I). This compound inhibits the activity of phase-I enzymes, such as cytochrome P450 enzymes. It also promotes the production of reactive oxygen species, regulates the cell cycle and serves as a robust anticancer component derived from various vegetables $(15,19,20)$. Sulforaphene exerts anticancer effects on lung, esophageal, colon, gastric, liver, breast, cervical and thyroid cancer by inducing apoptosis and blocking the cell cycle (19-26). Moreover, sulforaphane and sulforaphene possess similar structures, anticancer mechanisms and inhibitory effects (promoting cancer cell apoptosis and inhibiting the cell cycle) on colon cancer progression (15-17,20). However, their underlying mechanisms 
of action are different. In addition, the two compounds are effective on different types of cancer and it is unknown whether they can complement each other or act synergistically. Therefore, the aim of the present study was to assess the effects of sulforaphane and sulforaphene on colon cancer cells and examine on the differences in gene regulation mediated by these two compounds.

\section{Materials and methods}

Materials. The human colorectal adenocarcinoma cell line, SW480, was purchased from the American Type Culture Collection (CCL-228 $8^{\mathrm{TM}}$ ) and cultured in DMEM/high glucose medium (HyClone; Cytiva; cat. no. SH30022.01B) containing $10 \%$ fetal bovine serum (HyClone; Cytiva; cat. no. $\mathrm{SH} 30087.01)$ at $37^{\circ} \mathrm{C}$ in a $5 \% \mathrm{CO}_{2}$ incubator. Sulforaphane (Abcam; cat. no. ab141970) and sulforaphene (Abcam; cat. no. ab141972) were purchased from Abcam (purity $>98 \%$ ), solubilized in double-distilled water and diluted to $25 \mu \mathrm{mol} / 1$ in culture medium. The RNA TRIzol ${ }^{\circledR}$ extraction kit was purchased from Invitrogen, Thermo Fisher Scientific, Inc.. The GN-genechip Clariom ${ }^{\mathrm{TM}} \mathrm{S}$ Array next-generation sequencing (NGS) chip was purchased from Affymetrix, Thermo Fisher Scientific, Inc. (cat. no. 902927; human).

Comparison of physical and chemical properties. Based on the reagents sulforaphane and sulforaphene presented on the supplier's website (sulforaphane: https://www. abcam.cn/s-sulforaphane-ah-receptor-antagonist-ab141970.html; sulforaphene: https://www.abcam.cn/s-sulforaphene-carcinogenesis-inhibitor-ab141972.html), the physicochemical properties (e.g. molecular structure, chemical name, molecular mass, form, purity and solubility) of the two compounds were compared and compiled. Literature surrounding their biological activity was reviewed in order to evaluate the different effects of the two compounds on cancer, from a physicochemical perspective.

Cell treatment. SW480 colon cancer cells were maintained in DMEM/high glucose medium containing $10 \%$ fetal bovine serum, at $37^{\circ} \mathrm{C}$ in a $5 \% \mathrm{CO}_{2}$ incubator, and were cultured to $>90 \%$ confluence. A total of three groups were used, namely sulforaphane, sulforaphene and blank control. The cells were treated at the logarithmic growth phase, and each group was plated in triplicate.

Measurement of cell viability. $\mathrm{IC}_{50}$ values were determined by assessing cell viability at $48 \mathrm{~h}$, using the MTT method (Fig. S1). Drug treatment was performed using $25 \mu \mathrm{mol} / 1$ sulforaphane or sulforaphene. An equivalent volume (10 $\mu \mathrm{l}$ in a $100 \mu \mathrm{l}$ culture system) of double-distilled water was added to the blank control group. Formazan was dissolved in dimethyl sulfoxide, and the OD value was measured at $490 \mathrm{~nm}$.

$N G S$. Following $48 \mathrm{~h}$ of incubation, total RNA was extracted using TRIzol ${ }^{\circledR}$ reagent (Thermo Fisher Scientific). The quality of the RNA was assessed using a Nanodrop ${ }^{\text {TM }} 2000$ spectrophotometer (Thermo Fisher Scientific, Inc.) and an Agilent 2100 Bioanalyzer (Agilent Technologies Inc.). The samples met the following conditions prior to NGS: i) A260/A280 ratio, 1.7-2.2; ii) RNA integrity number $\geq 7.0$; and iii) $28 \mathrm{~S} / 18 \mathrm{~S}$ ratio $>0.7$.
Biotinylated cRNAs were prepared from $6 \mu \mathrm{g}$ total RNA according to the standard Affymetrix protocol (Expression Analysis Technical Manual, 2001, Affymetrix) (27). Following fragmentation, $10 \mu \mathrm{g}$ of cRNA were hybridized for $16 \mathrm{~h}$ at $45^{\circ} \mathrm{C}$ on GN-GeneChip Clariom S Array, human (Thermo Fisher Scientific; cat. no. 902926). GeneChips were washed and stained in the GeneChip Fluidics Station 450 (Thermo Fisher Scientific, Inc.) and scanned using the GeneChip Scanner 3000 (Thermo Fisher Scientific, Inc.). Paired-end sequencing was performed, resulting in short sequence reads of $\sim 150 \mathrm{bp}$.

Data analysis. The data were analyzed with Microarray Suite version 5.0 (MAS 5.0) using Affymetrix default analysis settings and global scaling as the normalization method. The trimmed mean target intensity of each array was arbitrarily set to 100 . Pearson's correlation and principal component analysis were performed on the NGS data to determine the homogeneity of the three replicate samples in the group. Data passed the quality control threshold if the correlation coefficient was $>0.8$ with 'cor()' and 'princomp()'. The limma package (version 3.40.2; http://www.bioconductor. org/packages/release/bioc/html/limma.html) was used to analyze differential gene expression between the groups, and differentially expressed genes were defined as fold-change $\geq 2.0$, false discovery rate $<0.05, \mathrm{P}<0.05$ and adjusted $\mathrm{P}<0.05$. Based on these results, differential gene and cluster analyses were performed and the data were evaluated by Gene Ontology (GO) analysis and Kyoto Encyclopedia of Genes and Genomes (KEGG) pathway enrichment analysis using the DAVID 6.8 database (https://david.ncifcrf.gov/). The RIdeogram package was used to map the location of differentially expressed genes across the chromosomes (https://cran.r-project. org/web/packages/RIdeogram/index.html). All statistical analyses were carried out using $\mathrm{R}$ software version 3.5.1 (https://www.r-project.org/).

\section{Results}

Comparison of the differences in the physicochemical properties of sulforaphane and sulforaphene. Sulforaphane and sulforaphene are both single-chain compounds derived from cruciferous vegetables and share the same chiral center, resulting in the formation of specific isomers (15). Interestingly, sulforaphane [Chemical Abstracts Service (CAS) no. 155320-20-0] and sulforaphene (CAS no. 592-95-0) are distinguished by the presence of only one carbon-carbon double bond. The physicochemical properties of the two compounds with regard to their molecular structure, chemical name, molecular mass, form, purity and solubility were evaluated in order to identify the specific differences between them (Table I). Notably, sulforaphane and sulforaphene underwent specific changes in soluble solvents, resulting in a $2 \mathrm{~g} / \mathrm{mol}$ difference in molecular mass. Based on the XLogP3 values, sulforaphene exhibited a higher lipid-water partition coefficient. Its solubility in organic solvents has not been previously examined, as opposed to sulforaphane, which exhibits a slightly lower XLogP3 value and is soluble in ethanol and DMSO. In addition, the carbon-carbon double bonds resulted in a reduced number of rotatable bonds in sulforaphene. 
Table I. Differences between sulforaphane and sulforaphene.

\begin{tabular}{|c|c|c|}
\hline Property & Sulforaphane & Sulforaphene \\
\hline Molecular formula & $\mathrm{C}_{6} \mathrm{H}_{11} \mathrm{NOS}_{2}$ & $\mathrm{C}_{6} \mathrm{H}_{9} \mathrm{NOS}_{2}$ \\
\hline \multicolumn{3}{|l|}{ Structural formula } \\
\hline Chemical name & $\begin{array}{l}\text { 1-isothiocyanato-4-[(S)-methylsulfinyl] } \\
\text { butane }\end{array}$ & $\begin{array}{l}\text { (E)-4-isothiocyanato-1-methylsulfinylbut- } \\
\text { 1-ene }\end{array}$ \\
\hline Molecular mass, $\mathrm{g} / \mathrm{mol}$ & 177.3 & 175.264 \\
\hline SMILES & $\mathrm{C}[\mathrm{S} @](=\mathrm{O}) \mathrm{CCCCN}=\mathrm{C}=\mathrm{S}$ & $\mathrm{CS}(=\mathrm{O}) \mathrm{C}=\mathrm{CCCN}=\mathrm{C}=\mathrm{S}$ \\
\hline CAS Number & $155320-20-0$ & $592-95-0$ \\
\hline Purity, \% & $>98$ & $>98$ \\
\hline Form/State & Solid & Solid \\
\hline Solubility & $\begin{array}{l}\text { Soluble in water, in ethanol and } \\
\text { in DMSO }\end{array}$ & Soluble in water \\
\hline XLogP3 & 1.4 & 1.5 \\
\hline Number of hydrogen bond donors & 0 & 0 \\
\hline Number of hydrogen bond acceptors & 4 & 4 \\
\hline Number of rotatable bonds & 5 & 4 \\
\hline Source & Brassica oleracea & Brassica oleracea \\
\hline Biological characterization & $\begin{array}{l}\text { Non-competitive antagonist of the } \\
\text { aryl hydrocarbon receptor, natural } \\
\text { isothiocyanate, antitumor agent, active } \\
\text { in vivo and in vitro (https://www.abcam. } \\
\text { cn/s-sulforaphane-ah-receptor-antagonist- } \\
\text { ab141970.html) }\end{array}$ & $\begin{array}{l}\text { Carcinogenesis inhibitor, anticancer, } \\
\text { antidiabetic and antioxidant effects, active } \\
\text { in vivo and in vitro } \\
\text { (https://www.abcam.cn/s-sulforaphene- } \\
\text { carcinogenesis-inhibitor-ab141972.html) }\end{array}$ \\
\hline Type of anti-cancer effect & 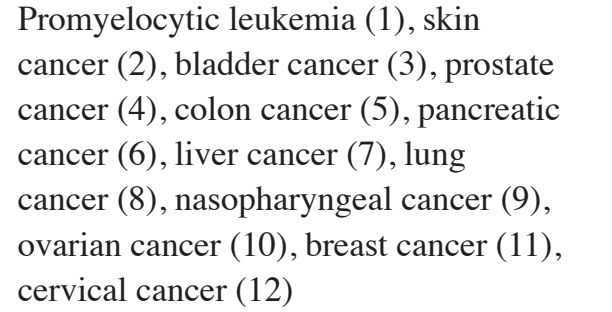 & $\begin{array}{l}\text { Lung cancer (21), esophageal cancer (20), } \\
\text { colon cancer (22), gastric cancer (23), liver } \\
\text { cancer (24), breast cancer (19), cervical } \\
\text { cancer (25), thyroid cancer (26) }\end{array}$ \\
\hline
\end{tabular}

CAS, Chemical Abstracts Service; SMILES, simplified molecular-input line-entry system.
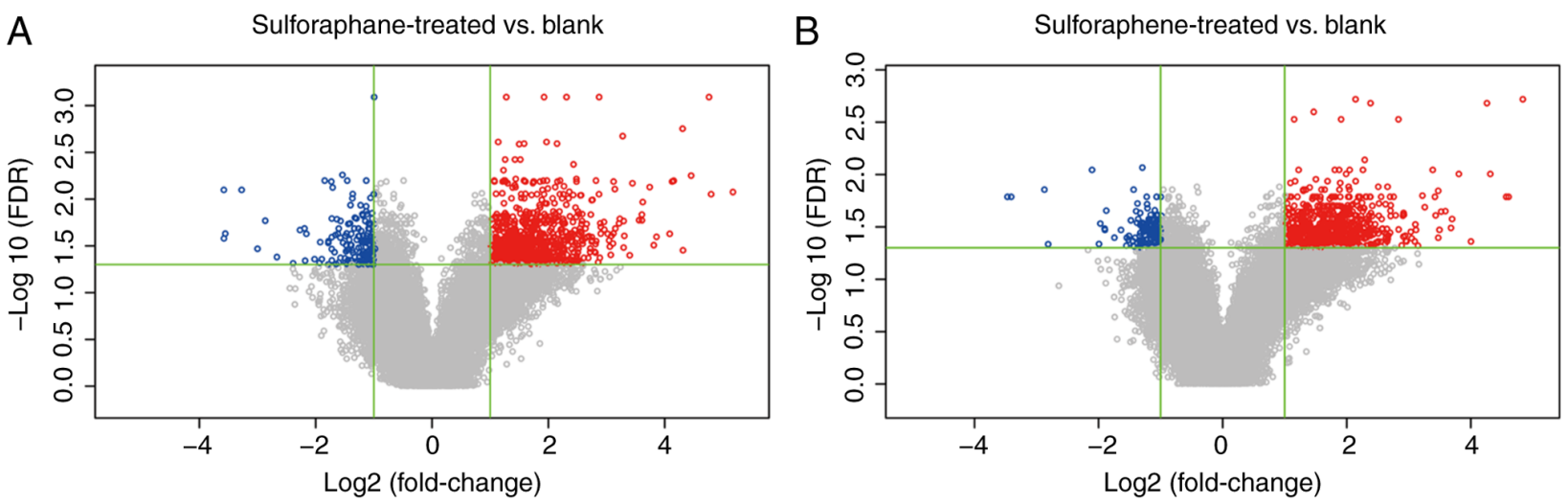

Figure 1. Volcano plot of differentially expressed genes following SW480 cell treatment with sulforaphane and sulforaphene. (A) Sulforaphane-treated vs. blank. (B) Sulforaphene-treated vs. blank. Each symbol represents a gene. Red symbols indicate an increase in gene expression following treatment, whereas blue symbols denote a decrease in gene expression following treatment. The gray symbols indicate a change in gene expression that is not statistically different. FDR, false discovery rate. 
A

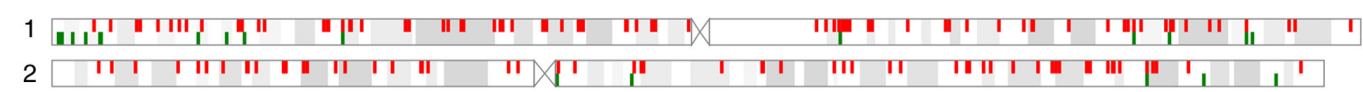

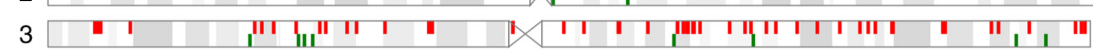

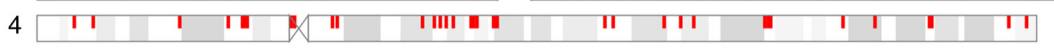

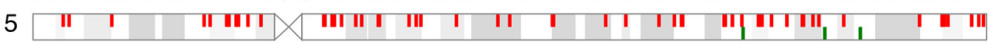

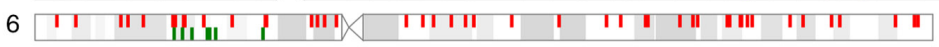

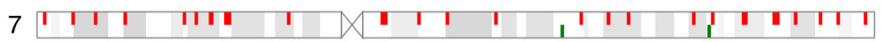

8 1 1 | |

2 \begin{tabular}{|l|l|l|l|l|l|l|l|l|l|l|l|l|}
\hline 11 & 1
\end{tabular}

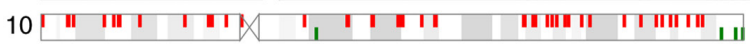

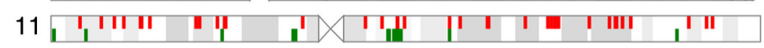

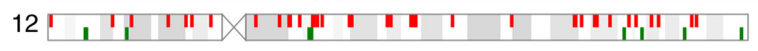

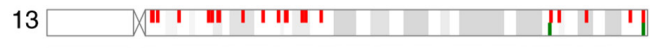

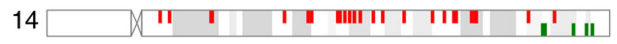

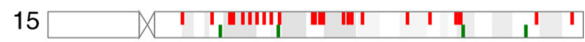

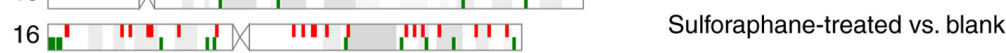

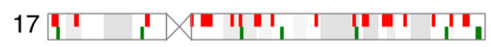

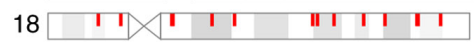

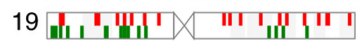

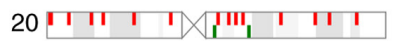

$21 \square \times \square+111111$

$22 \square$ XII II! ",

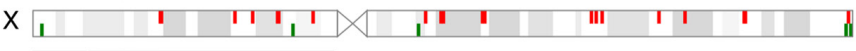

$\mathrm{Y}+\mathrm{P}$

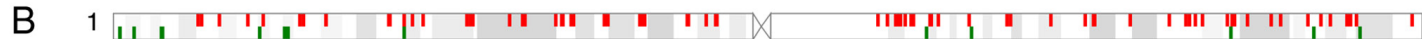

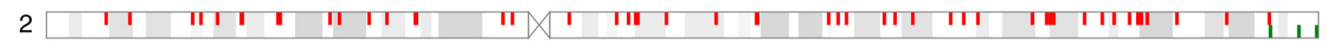

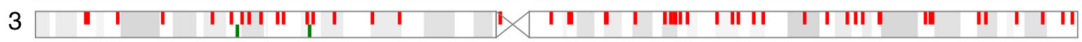

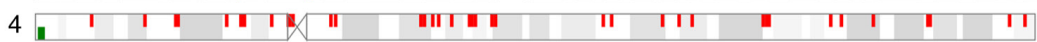

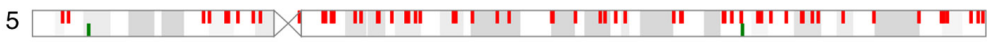

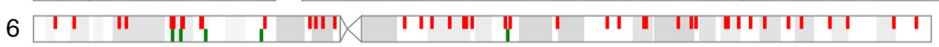

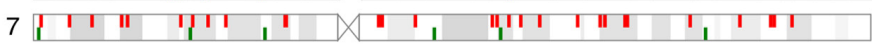

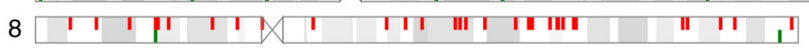

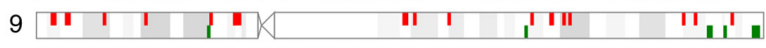

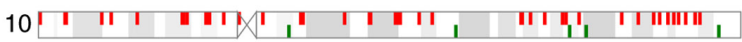

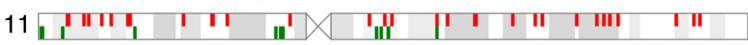

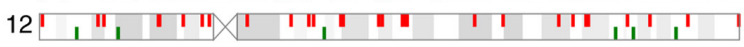

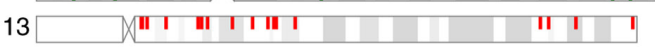

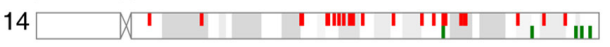

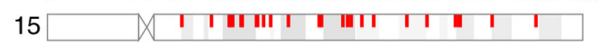

16 II II I II III II II I II, Sulforaphene-treated vs. blank

17 |

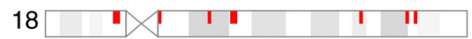

19 औ

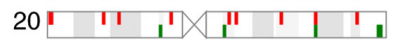

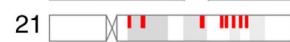

$22 \square+\left(\begin{array}{llll}\square & 1\end{array}\right.$

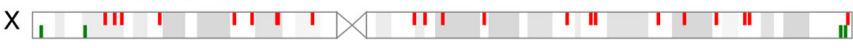

Y

Figure 2. Chromosomal distribution of differentially expressed genes following SW480 cell treatment with sulforaphane and sulforaphene. (A) Sulforaphane-treated vs. blank. (B) Sulforaphene-treated vs. blank. Each dot represents a gene, with red dots indicating an increase in gene expression after treatment, blue dots indicating a decrease in gene expression after treatment.

RNA and chip assay quality control. The viability of the cells was measured after $48 \mathrm{~h}$ of treatment with the two compounds, at a concentration of $25 \mu \mathrm{mol} / 1$, and the results showed that both reached effective inhibitory concentrations (Fig. S1). The quality of the total RNA extracted using the TRIzol ${ }^{\circledR}$ method was assessed in each sample (Table SI). The signal intensity of the sequencing chip probes for each sample was tested and analyzed using Pearson's correlation and principal component analyses. The results indicated that the correlation coefficient for each sample pair was $>0.85$ in three treatment groups 
A
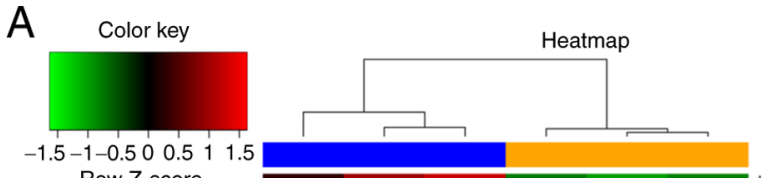

Row Z-score

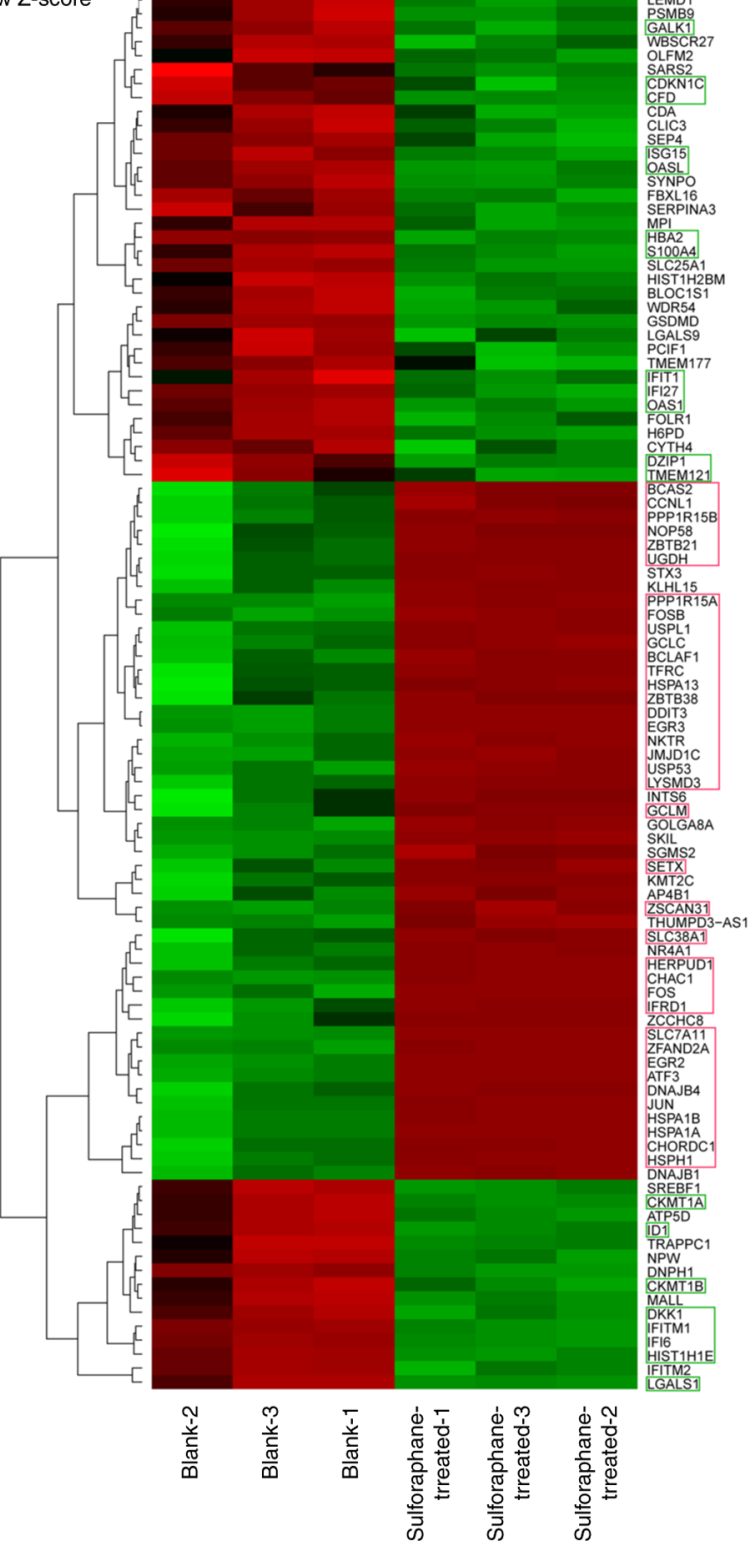

B

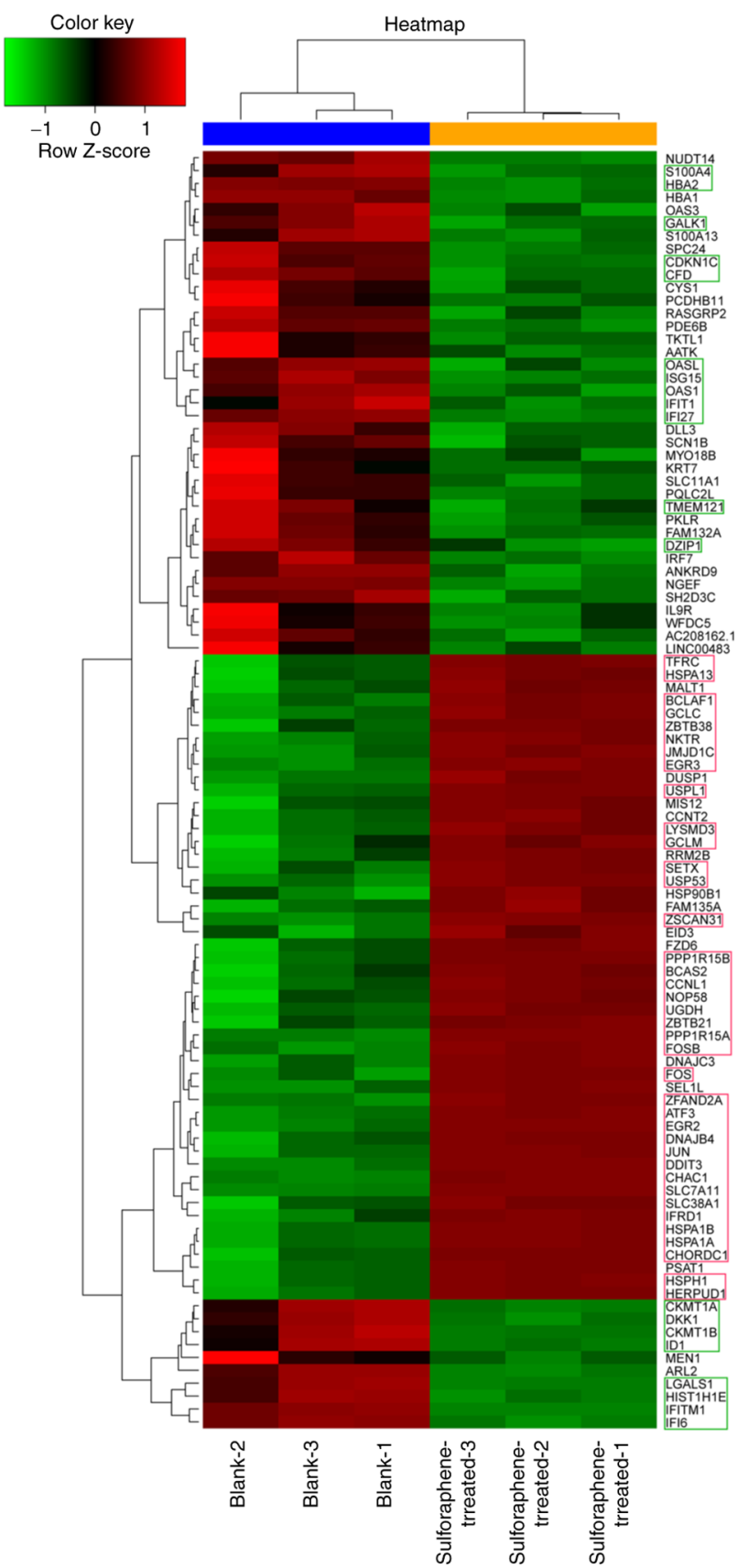

Figure 3. Hierarchical clustering analysis of differentially expressed genes following SW480 cell treatment with sulforaphane and sulforaphene. (A) Sulforaphane-treated vs. blank. (B) Sulforaphene-treated vs. blank. Red represents an increase in gene expression following treatment, whereas green denotes a decrease. The genes that changed consistently after treatment with both compounds are highlighted in red and green boxes.

(Fig. S2). In the principal component 1 and principal component 2 dimensions, the aggregation and separation trends of the samples were distinct in the three groups (Fig. S3).

Differentially expressed gene analysis of the sulforaphene and sulforaphane groups. A total of 873 probes were differentially expressed in the sulforaphene group compared with the blank control group and 862 genes were obtained, of which 130 were downregulated and 732 were upregulated in the sulforaphene group. A total of 959 probes in the sulforaphane group were differentially expressed compared with the blank control group, and 949 genes were obtained. Among these, 161 genes were downregulated and 788 genes were upregulated in the sulforaphane group (Fig. 1). In addition, differential gene expression analysis was performed, and no genes were differentially expressed between the three groups.

Gene position distribution and cluster analysis of differential expression between the two groups. The genetic loci of the genes which were differentially expressed in the sulforaphene group were identified. The distribution position and number of differentially expressed genes on the 22 pairs of autosomes and the $\mathrm{X}$ and $\mathrm{Y}$ sex chromosomes did not markedly differ (Fig. 2).

Hierarchical clustering analysis indicated that the clustering of 58 genes (about $3 \%$ of the total differentially expressed 
A

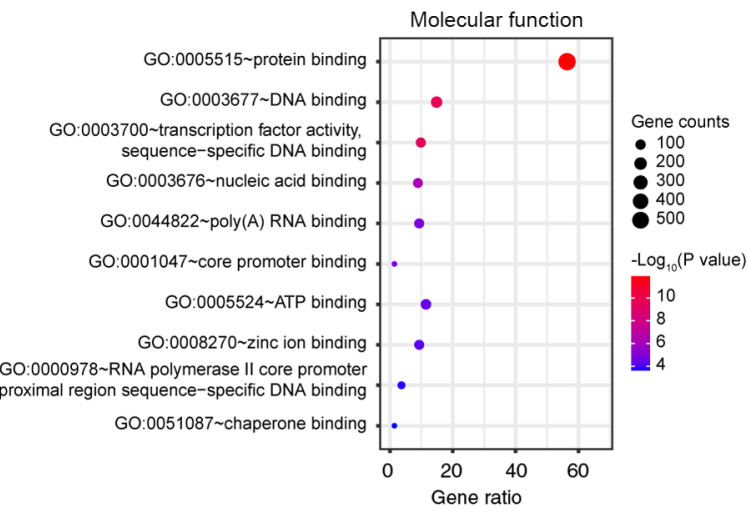

C

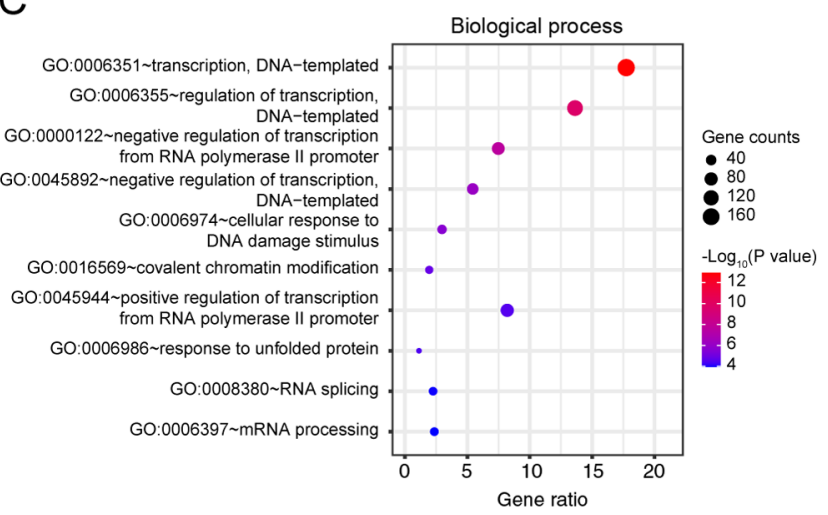

$\mathrm{E}$

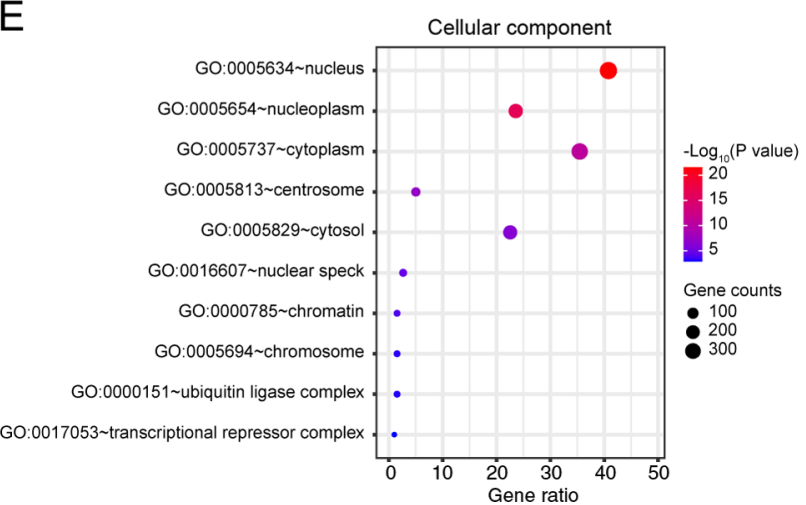

B

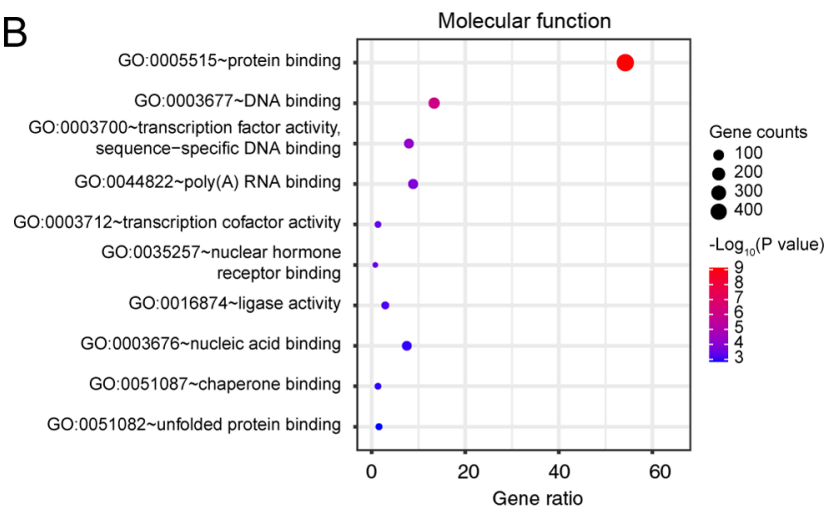

$\mathrm{D}$

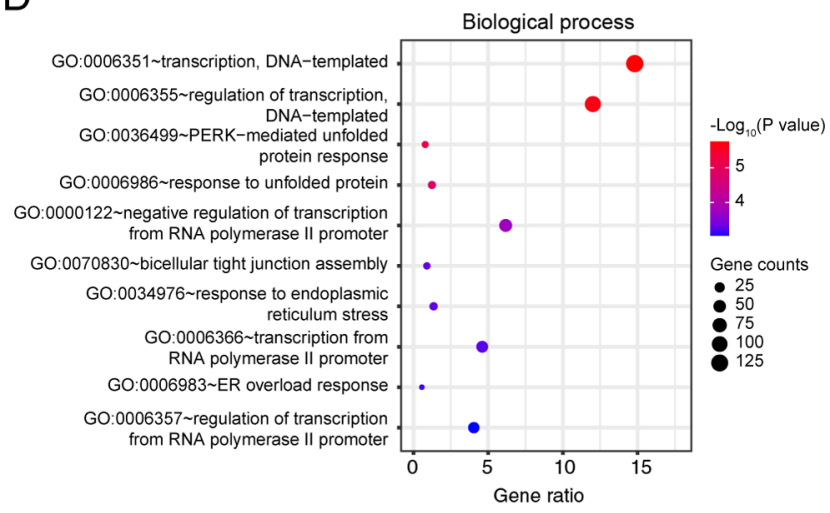

$\mathrm{F}$

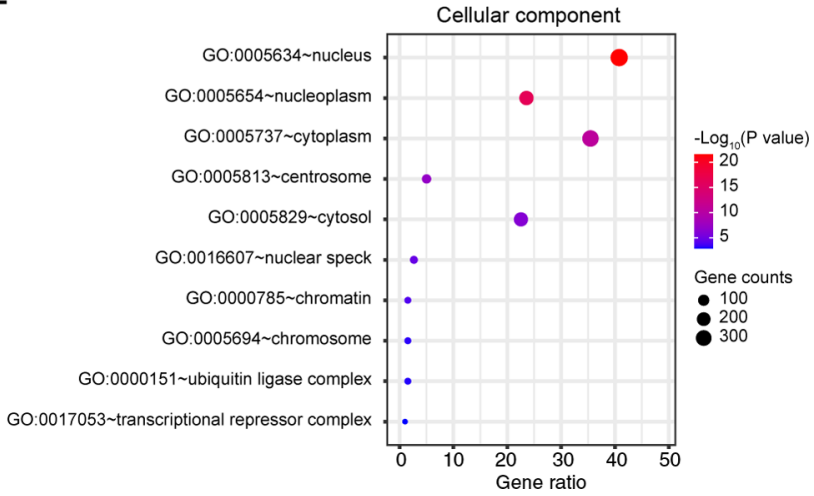

Figure 4. GO enrichment analysis. (A and B) Molecular function GO terms associated with (A) sulforaphane or (B) sulforaphene treatment. (C and D) Biological process GO terms associated with (C) sulforaphane or (D) sulforaphene treatment. Cellular component GO terms associated with (E) sulforaphane or (F) sulforaphene treatment. The size of the symbols represents the number of genes annotated under this term, whereas the color represents the P-value. GO, Gene Ontology.

genes) was similar between the two groups. A total of 20 genes (GALK1, OAS1, CKMTIA, DKK1, CKMT1B, IDI, LGALS1, HISTIHIE, IFITM1, IFI6, SIOOA4, HBA2, CDKNIC, CFD, OASL, ISG15, IFIT1, IFI27 TMEM121 and DZIPI) exhibited lower $\mathrm{Z}$ scores than the blank control group. The $\mathrm{Z}$ scores of the remaining 38 genes (BCAS2, CCNL1, PPP1R15B, NOP58, ZBTB21, UGDH, PPP1R15A, FOSB, USPL1, GCLC, BCLAF1, TFRC, HSPA13, ZBTB38, DDIT3, EGR3, NKTR, JMJDIC, USP53, LYSMD3, GCLM, SETX, ZSCAN31, SLC38AC, HERPUD1, CHAC1, FOS, IFRD1, SLC7A11, ZFAND2A, EGR2, ATF3, DNAJB4, JUN, HSPA1B, HSPAIA, CHORDC1 and $H S P H 1$ ) were higher than those noted in the blank control group (Fig. 3).

GO analysis. GO analysis of differentially expressed genes was performed using the DAVID 6.8 database. Gene enrichments were identified with regard to molecular function, biological processes and cellular components (Fig. 4). The majority of the genes in the sulforaphane and the sulforaphene-treated groups were enriched in the same functions, relative to the blank group, notably in 'protein binding' (GO:0005515, Fig. 4A and B), 'transcription, DNA-templated' (GO:0006351) and 'regulation of transcription, DNA-templated' (GO:0006355, Fig. 4C and D), 'nucleus' (GO:0005634) and 'nucleoplasm' (GO:0005654, Fig. 4E and F). However, subtle differences were noted in 'ATP binding' (GO:0005524, in sulforaphane group, Fig. 4A), 'negative regulation of transcription, DNA-templated' (GO:0045892, in sulforaphane group, Fig. 4A) and 'positive regulation of transcription from RNA polymerase II promoter' (GO:0045944, in sulforaphane group, Fig. 4A). Under 'cellular component', the two groups of annotations to terms are the same, while the unique sulforaphene group is 'transcription 
A

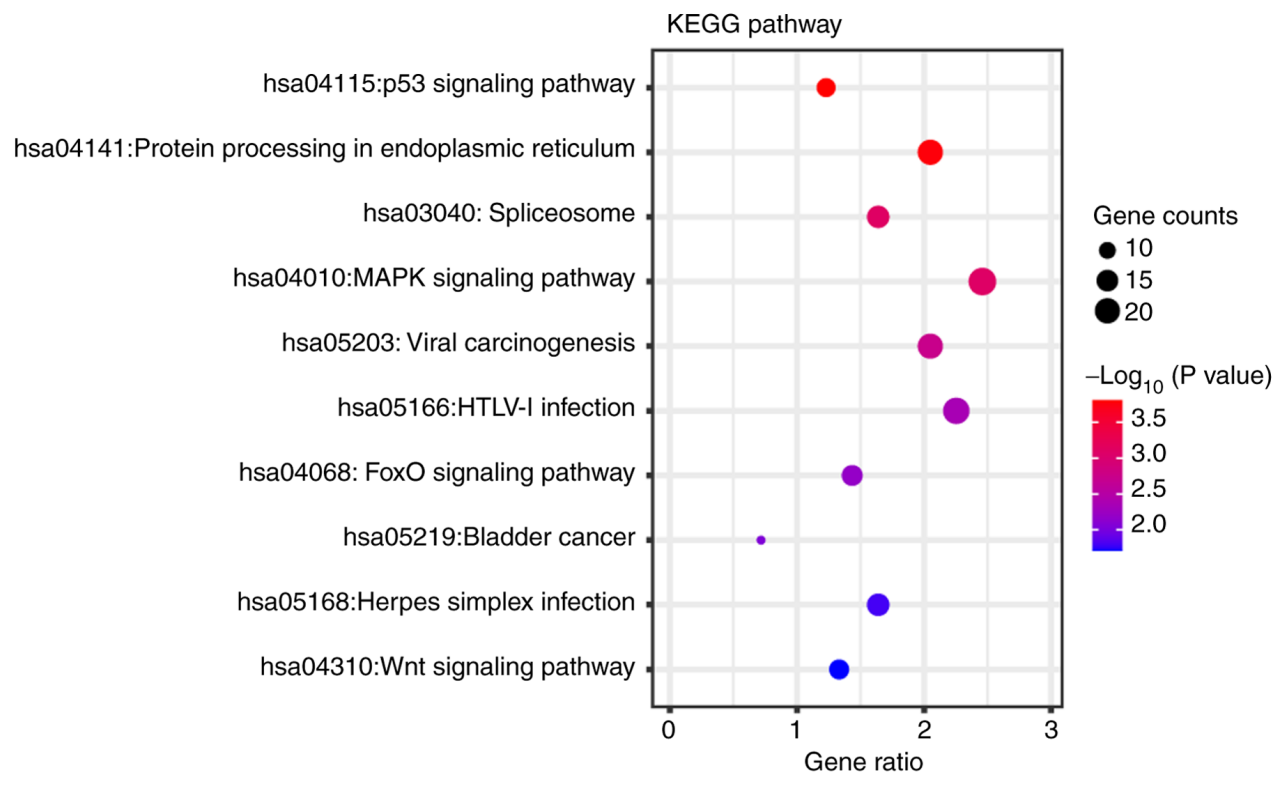

B

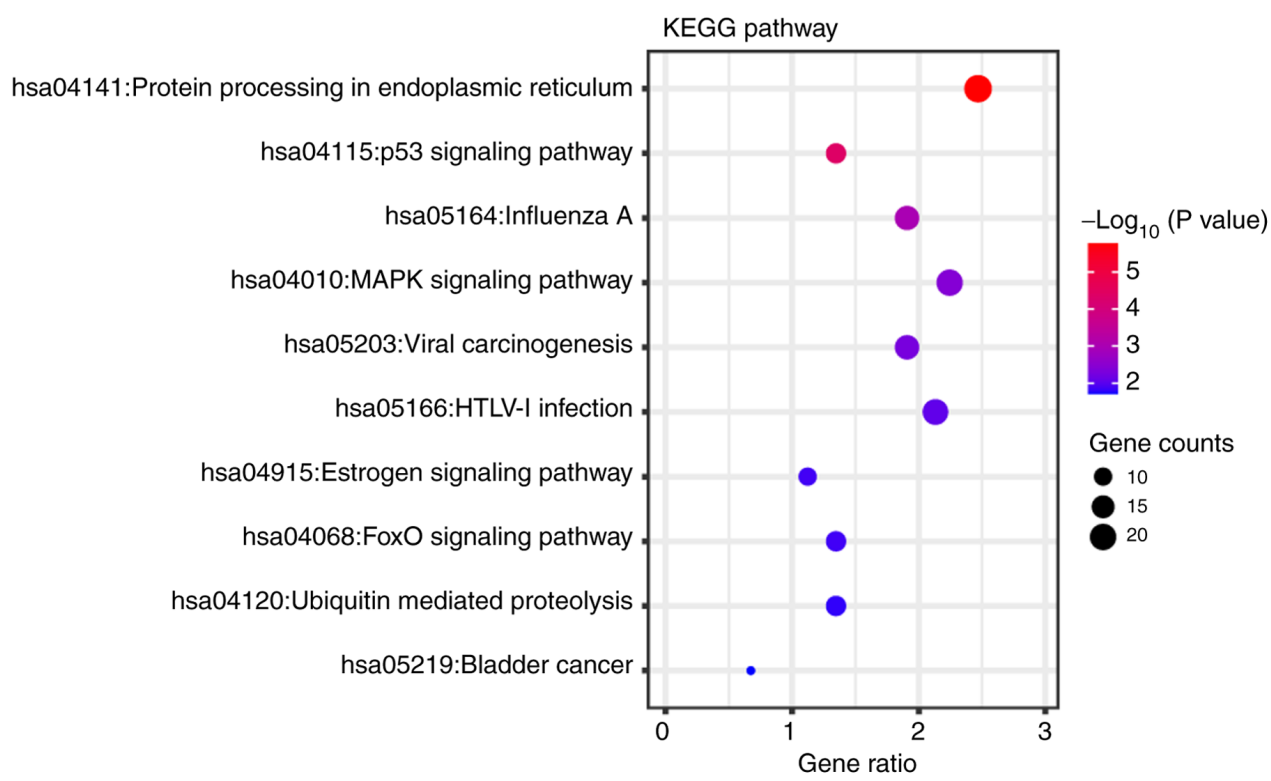

Figure 5. KEGG pathway annotation. (A) Sulforaphane-treated; (B) Sulforaphene-treated. The size of the symbols represents the number of genes annotated under this term, whereas the color represents the P-value. KEGG, Kyoto Encyclopedia of Genes and Genomes.

cofactor activity' (GO:003712, Fig. 4B) and 'transaction from RNA polymerase II promoter' (GO:0006366, Fig. 4D).

KEGG pathway annotations. The differentially expressed genes identified in the sulforaphane and the sulforaphene-treated groups were annotated using KEGG to assess the specific effects of these compounds on SW480 cells (Fig. 5). Overlapping pathways were identified, such as 'p53 signaling pathway' (hsa:04115), 'protein processing in endoplasmic reticulum' (hsa:04141), 'spliceosome' (hsa:03040), 'MAPK signaling pathway' (hsa:04010), 'viral carcinogenesis' (hsa:05203), 'HTLV-I infection' (hsa:05166), 'FOXO signaling pathway' (hsa:04068) and 'bladder cancer' (hsa:05219). In the sulforaphane group, the differentially expressed genes were uniquely found to be associated with the 'spliceosome' (hsa:03040), 'herpes simplex infection' (hsa:05168) and 'Wnt signaling pathway' (hsa:04310), while in the sulforaphene group the differentially expressed genes were associated with the 'estrogen signaling pathway' (hsa:04915) and 'ubiquitin mediated proteolysis' (hsa:04120). What's more, a deeper visualization of the pathways regulated by these two compounds are displayed (Fig. S4). In the p53 signaling pathway, both compounds upregulated MDM2 (Fig. S4A and B), while in the MAPK signaling pathway both upregulated RafB (Fig. S4C and D). The FOXO pathway was inhibited by sulforaphene, and sulforaphane directly upregulated $F O X O$ (Fig. S4E and F). The WNT pathway was up-regulated by sulforaphane and core molecules such as Wnt and $A P C$ (Fig. S4G), while sulforaphene can upregulate some members of the estrogen signaling pathway (Fig. S4H).

\section{Discussion}

Colorectal cancer is one of the most common gastrointestinal malignancies. The Global Cancer Observatory 2018 data suggested that the incidence of colon cancer ranked third 
among men and second among women (28). In 2018, a total of 1,096 million new cases and 551,000 deaths from colorectal cancer were reported (28). The National Cancer Center of China released the status of malignant tumors in 2016, which stated that the number of new patients and deaths from colorectal cancer ranked fourth among other cancer types, accounting for 376,000 and 191,000 new cases, respectively (29). Therefore, the identification of practical and effective therapeutic drugs has become an urgent medical requisite. Recent years have witnessed the development of new technologies and treatment methods in the field of cancer research. Moreover, the application of NGS has facilitated research into the pathogenesis of colorectal cancer.

Cruciferous plants, such as cabbage, broccoli, cauliflower, kale, lettuce and white radish contain $>120$ types of glucosinolates that are similar in structure, consisting of one $\beta-D$ glucosinolate, one sulfonated oxime group and a side chain. When glucosinolates are hydrolyzed by acid or specific enzymes (such as myrosinase), they produce structural homologs and isothiocyanates (14-16). Previous studies have shown that isothiocyanates can improve the immunity of the body and enhance antioxidant (in the liver), anti-inflammatory (in the prostate, oral cavity and glial cells) and anticancer (in the skin, bladder, colon, pancreas, liver, lung, nasopharynx, ovary, breast, esophagus, stomach, cervix and thyroid) activities (2-11,19,20,23-26,30,31). Sulforaphene is the isothiocyanate with the most potent anticancer ingredients found in vegetables (15). Phase-I and -II enzymes are involved in the metabolism of chemical carcinogens. Phase-I enzymes activate chemical carcinogens to electrophiles, whereas phase-II enzymes convert electrophiles to low-toxicity metabolites and readily excreted substances (15). Sulforaphene inhibits phase-I enzymes, such as cytochrome P450 enzymes and induces the expression levels of phase-II detoxification enzymes, such as glutathione sulfur transferase, epoxide hydrolase and uridine 5'-diphospho-glucuronosyltransferase-glucuronyltrans-

ferase (15). Due to the lipophilic nature of sulforaphene, the inhibition of the biosynthesis of phospholipids and other lipid substances is a vital mechanism that can induce growth arrest and apoptosis of cancer cells (32-34).

Previous studies have shown that sulforaphane and sulforaphene have the same active center and similar chemical structures (15-17). Despite their similarities, they have been reported to be effective against distinct cancer types, as aforementioned. This may be attributed to the limited number of studies performed on these two compounds or could be due to their limited efficacy against certain cancer types.

In the present study, the effects of sulforaphane and sulforaphene were evaluated in colorectal cancer cells using NGS in order to assess the differences between the anticancer efficacy of the two compounds. Sulforaphene increased and decreased the expression levels of 732 and 130 genes, respectively, while sulforaphane increased and decreased the expression levels of 788 and 161 genes, respectively. Notably, none of these differentially expressed genes were simultaneously detected in all three groups, which indicated that sulforaphene and sulforaphane exhibited distinct roles in colon cancer cells. However, these effects need to be confirmed using multiple colon cancer cell lines. Although the SW480 cell line is widely for the study of colon cancer, it still does not fully represent the physiological characteristics of colon cancer, and it is imperative to validate it using a wider variety of colon cancer cell lines or by extracting primary cells from tumor tissues of colon cancer patients in a sufficient sample size. This is one of the limitations of the present study. Hierarchical clustering analysis of differentially expressed genes indicated that the $\mathrm{Z}$ score of 20 genes, such as $G A L K 1$, was lower than that of the control group, whereas the cluster analysis of the 38 genes, such as $B C A S 2$, was higher than that of the control group. This was observed in both sulforaphene and sulforaphane groups. These 58 genes may be the common targets of sulforaphane and sulforaphene. Sulforaphene and sulforaphane may induce anticancer effects by affecting these 58 genes. GO and KEGG analyses indicated that the functions and pathways enriched by the differentially expressed genes produced by sulforaphene and sulforaphane were similar. These pathways and functions included 'protein binding' (GO:0005515), 'transcription, DNA-templated' (GO:0006351), 'regulation of transcription, DNA-templated' (GO:0006355), 'nucleus' (GO:0005634), 'nucleoplasm' (GO:0005654), 'p53 signaling pathway' (hsa:04115), 'MAPK signaling pathway' (hsa:04010) and 'FOXO signaling pathway' (hsa:04068), which are common cancer-associated functions or pathways. Sulforaphane may also act on the Wnt pathway, suggesting a potential role in epithelial-to-mesenchymal transition (9). Furthermore, sulforaphene is specifically enriched in the estrogen signaling pathway, indicating that it is associated with female endocrine cancers, such as those of breast, cervical, endometrial and ovarian origin, which is consistent with previous reports $(19,25,35,36)$.

Based on the aforementioned results, it may be hypothesized that sulforaphane and sulforaphene exert similar anticancer effects. However, KEGG signaling pathway enrichment suggested differences between the two molecules. For example, genes with altered expression in both groups were enriched in the 'p53 signaling pathway', 'MAPK signaling pathway' and 'FOXO signaling pathway' pathways. p53 is a key transcription factor required in maintaining DNA integrity and is often referred to as the 'guardian of the genome'. Under steady-state conditions, the activity of p53 in colon cancer cells is inhibited by its binding to the negative regulators mouse double minute (MDM) 2 and MDM4, which induce p53 ubiquitination and protein degradation, while maintaining the expression of tumor suppressors at low levels in order to circumvent their tumor inhibitory function (37). The oncogenic activity of sulforaphane and sulforaphene was presumably caused by the upregulation of MDM2 expression. The MAPK/PI3K signaling pathway is involved in cell proliferation and survival. Changes in the expression levels of proteins involved in this pathway cause an increase in the proliferative potential of tumor cells $(24,27,35,36)$. KRAS, BRAF and PIK3CA mutations are common in colorectal cancer (38). The oncogenic properties of sulforaphane and sulforaphene may be due to their ability to modulate RafB and MAPK kinase kinase 1 and consequently affect the MAPK signaling pathway. FOXO family members alter cell metabolism by inhibiting glycolysis and promoting mitochondrial respiration $(39,40)$, which is crucial for cancer cells with a more active metabolism. The results of KEGG pathway analysis demonstrated that sulforaphane and sulforaphene affected the expression 
levels of AMPK, PI3K and MDM2, which may in turn influence elements of the FOXO pathway. The Wnt/ $\beta$-catenin pathway regulates several cellular functions, such as stem cell regeneration and organogenesis (41). The activation of this pathway occurs at the bottom of intestinal crypts and is associated with the loss of function of adenomatous polyposis coli ( $A P C$ ), a tumor regulator (41). APC was one of the genes significantly upregulated by sulforaphane treatment. This may have been caused by changes in the expression of proteins associated with specific pathways, such as WNT and MAPK, which may also explain the differences in the anticancer efficacy of sulforaphane and sulforaphene. Nevertheless, the changes caused by sulforaphane on colon cancer crypts requires experimental validation using immunohistochemical analysis and pathological evaluation. The unique pathway for sulforaphene identified was the 'ubiquitin-mediated proteolysis' pathway, which may be associated with ubiquitination and degradation of the p53 protein (37). In contrast to sulforaphane, sulforaphene may regulate the 'estrogen signaling pathway' through the regulation of genes, such as heparin-binding-EGF, which is related to previous studies suggesting an association with breast and cervical cancers $(19,25)$. However, the effects of sulforaphene on ovarian and endometrial cancer require further investigation.

In the present study, only one colon cancer cell line was used. The genes presented in this study were only obtained using NGS and bioinformatics analysis and were not validated using quantitative PCR at the RNA level or western blot analysis at the protein level, which is the greatest limitation of the present study. Due to modifications, such as RNA $\mathrm{m}^{6} \mathrm{~A}$ methylation, the sequencing results may differ from the actual RNA and protein levels. Moreover, the analysis did not include functional tests to assess the proliferative, invasive and migratory activities of colon cancer cells treated with sulforaphane and sulforaphene. The conclusions relating to the anticancer effects of these two molecules are completely based on previous reports; therefore, bias is likely. Several studies have shown that different concentration levels of sulforaphane and sulforaphene exert specific anticancer activities $(15,19,23,25)$. However, whether the concentration used in the present study was optimal remains to be explored using a concentration gradient test.

In conclusion, sulforaphane and sulforaphene have similar biological activities and exert similar effects on colon cancer cells. However, a slight difference was observed in the functional annotation of the genes and in pathway enrichment analysis. Both of these compounds may affect the p53, MAPK and FOXO signaling pathways in colon cancer cells. Sulforaphane may be associated with the Wnt pathway, while sulforaphene with the estrogen pathway.

\section{Acknowledgements}

Not applicable.

\section{Funding}

This work was supported by The Research on Evaluation and Control Technology of Characteristic Agricultural
Products Quality Safety and Nutritional Function (grant no. 2017JHZ010).

\section{Availability of data and materials}

The datasets generated and/or analysed during the current study are available in the Gene Expression Omnibus under accession no. GSE174444 (https://www.ncbi.nlm.nih.gov/geo/query/acc. cgi?acc=GSE174444).

\section{Authors' contributions}

LG and JSW carried out the experiments. YHZ and JHL analyzed the experimental results. FYD, DC and XZ reviewed the results and graphed the experimental results. YTW and SQZ designed and managed the experiments. YTW and SQZ confirm the authenticity of all the raw data. FYD and JSW wrote the manuscript. All authors read and approved the final manuscript.

\section{Ethics approval and consent to participate}

Not applicable.

\section{Patient consent for publication}

Not applicable.

\section{Competing interests}

The authors declare that they have no competing interests.

\section{References}

1. Alhazmi N, Pai CP, Albaqami A, Wang H, Zhao X, Chen M, Hu P, Guo S, Starost K, Hajihassani O, et al: The promyelocytic leukemia protein isoform PML1 is an oncoprotein and a direct target of the antioxidant sulforaphane (SFN). Biochim Biophys Acta Mol Cell Res 1867: 118707, 2020.

2. Li S, Yang Y, Sargsyan D, Wu R, Yin R, Kuo HD, Yang I, Wang L, Cheng D, Ramirez CN, et al: Epigenome, transcriptome and protection by sulforaphane at different stages of UVB-induced skin carcinogenesis. Cancer Prev Res (Phila) 13: 551-562, 2020 .

3. Mastuo T, Miyata Y, Yuno T, Mukae Y, Otsubo A, Mitsunari K Ohba K and Sakai H: Molecular mechanisms of the anti-cancer effects of isothiocyanates from cruciferous vegetables in bladder cancer. Molecules 25: 575, 2020

4. Zhang Z, Garzotto M, Davis EW II, Mori M, Stoller WA, Farris PE, Wong CP, Beaver LM, Thomas GV, Williams DE, et al: Sulforaphane bioavailability and chemopreventive activity in men presenting for biopsy of the prostate gland: A randomized controlled trial. Nutr Cancer 72: 74-87, 2020.

5. Gasparello J, Gambari L, Papi C, Rozzi A, Manicardi A, Corradini R, Gambari R and Finotti A: High levels of apoptosis are induced in the human colon cancer HT-29 cell line by co-administration of sulforaphane and a peptide nucleic acid targeting miR-15b-5p. Nucleic Acid Ther 30: 164-174, 2020.

6. Desai P, Wang KZ, Ann D, Wang J and Prabhu S: Efficacy and pharmacokinetic considerations of loratadine nanoformulations and its combinations for pancreatic cancer chemoprevention. Pharm Res 37: 21, 2020.

7. Dos Santos PWDS, Machado ART, De Grandis RA, Ribeiro DL, Tuttis K, Morselli M, Aissa AF, Pellegrini M and Antunes LMG: Transcriptome and DNA methylation changes modulated by sulforaphane induce cell cycle arrest, apoptosis, DNA damage, and suppression of proliferation in human liver cancer cells. Food Chem Toxicol 136: 111047, 2020. 
8. Chen Y, Chen JQ, Ge MM, Zhang Q, Wang XQ, Zhu JY, Xie CF, Li XT, Zhong CY and Han HY: Sulforaphane inhibits epithelial-mesenchymal transition by activating extracellular signal-regulated kinase 5 in lung cancer cells. J Nutr Biochem 72: $108219,2019$.

9. Chen L, Chan LS, Lung HL, Yip TTC, Ngan RKC, Wong JWC, Lo KW, Ng WT, Lee AWM, Tsao GSW, et al: Crucifera sulforaphane (SFN) inhibits the growth of nasopharyngeal carcinoma through DNA methyltransferase 1 (DNMT1)/Wnt inhibitory factor 1 (WIF1) axis. Phytomedicine 63: 153058, 2019.

10. Tian M, Tian D, Qiao X, Li J and Zhang L: Modulation of Myb-induced NF-kB-STAT3 signaling and resulting cisplatin resistance in ovarian cancer by dietary factors. J Cell Physiol 234: 21126-21134, 2019.

11. Li S, Chen M, Wu H, Li Y and Tollefsbol TO: Maternal epigenetic regulation contributes to prevention of estrogen receptor-negative mammary cancer with broccoli sprout consumption. Cancer Prev Res (Phila) 13: 449-462, 2020.

12. Hussain A, Priyani A, Sadrieh L, Brahmbhatt K, Ahmed M and Sharma C: Concurrent sulforaphane and eugenol induces differential effects on human cervical cancer cells. Integr Cancer Ther 11: 154-165, 2012

13. Li D, Shao R, Wang N, Zhou N, Du K, Shi J, Wang Y, Zhao Z, Ye $\mathrm{X}$, Zhang $\mathrm{X}$ and $\mathrm{Xu} \mathrm{H}$ : Sulforaphane activates a lysosome-dependent transcriptional program to mitigate oxidative stress. Autophagy 17: 872-887, 2021.

14. Juge N, Mithen RF and Traka M: Molecular basis for chemoprevention by sulforaphane: A comprehensive review. Cell Mol Life Sci 64: 1105-1127, 2007.

15. Zhang Y and Talalay P: Mechanism of differential potencies of isothiocyanates as inducers of anticarcinogenic phase 2 enzymes. Cancer Res 58: 4632-4639, 1998.

16. Kamal MM, Akter S, Lin CN and Nazzal S: Sulforaphane as an anticancer molecule: Mechanisms of action, synergistic effects, enhancement of drug safety, and delivery systems. Arch Pharm Res 43: 371-384, 2020.

17. Clarke JD, Dashwood RH and Ho E: Multi-targeted prevention of cancer by sulforaphane. Cancer Lett 269: 291-304, 2008.

18. Xu Y, Han X, Li Y, Min H, Zhao X, Zhang Y, Qi Y, Shi J, Qi S, Bao Y and Nie G: Sulforaphane mediates glutathione depletion via polymeric nanoparticles to restore cisplatin chemosensitivity. ACS Nano 13: 13445-13455, 2019.

19. Pocasap P, Weerapreeyakul N and Thumanu K: Structures of isothiocyanates attributed to reactive oxygen species generation and microtubule depolymerization in HepG2 cells. Biomed. Pharmacother 101: 698-709, 2018

20. Zhang C, Zhang J, Wu Q, Xu B, Jin G, Qiao Y, Zhao S, Yang Y, Shang J, Li X and Liu K: Sulforaphene induces apoptosis and inhibits the invasion of esophageal cancer cells through MSK2/CREB/Bcl-2 and cadherin pathway in vivo and in vitro. Cancer Cell Int 19: 342, 2019.

21. Yang M, Wang H, Zhou M, Liu W, Kuang P, Liang H and Yuan Q: The natural compound sulforaphene, as a novel anticancer reagent, targeting PI3K-AKT signaling pathway in lung cancer. Oncotarget 7: 76656-76666, 2016.

22. Byun S, Shin SH, Park J, Lim S, Lee E, Lee C, Sung D, Farrand L, Lee SR, Kim KH, et al: Sulforaphene suppresses growth of colon cancer-derived tumors via induction of glutathione depletion and microtubule depolymerization. Mol Nutr Food Res 60: 1068-1078, 2016

23. Mondal A, Biswas R, Rhee YH, Kim J and Ahn JC: Sulforaphene promotes $\mathrm{Bax} / \mathrm{Bcl} 2$, MAPK-dependent human gastric cancer AGS cells apoptosis and inhibits migration via EGFR, p-ERK1/2 down-regulation. Gen Physiol Biophys 35: 25-34, 2016.

24. Pawlik A, Wała M, Hać A, Felczykowska A and Herman-Antosiewicz A: Sulforaphene, an isothiocyanate present in radish plants, inhibits proliferation of human breast cancer cells. Phytomedicine 29: 1-10, 2017.

25. Biswas R, Mondal A, Chatterjee S and Ahn JC: Evaluation of synergistic effects of sulforaphene with photodynamic therapy in human cervical cancer cell line. Lasers Med Sci 31: 1675-1682, 2016.
26. Chatterjee S, Rhee Y, Chung PS, Ge RF and Ahn JC: Sulforaphene enhances the efficacy of photodynamic therapy in anaplastic thyroid cancer through Ras/RAF/MEK/ERK pathway suppression. J Photochem Photobiol B 179: 46-53, 2018.

27. Baechler EC, Batliwalla FM, Karypis G, Gaffney PM, Moser K, Ortmann WA, Espe KJ, Balasubramanian S, Hughes KM, Chan JP, et al: Expression levels for many genes in human peripheral blood cells are highly sensitive to ex vivo incubation. Genes Immun 5: 347-353, 2004.

28. Bray F, Ferlay J, Soerjomataram I, Siegel RL, Torre LA and Jemal A: Global cancer statistics 2018: GLOBOCAN estimates of incidence and mortality worldwide for 36 cancers in 185 countries. CA Cancer J Clin 68: 394-424, 2018.

29. Chen W, Zheng R, Baade PD, Zhang S, Zeng H, Bray F, Jemal A, Yu XQ and He J: Cancer statistics in China, 2015. CA Cancer J Clin 66: 115-132, 2016

30. Ko MO, Kim MB and Lim SB: Relationship between chemical structure and antimicrobial activities of isothiocyanates from cruciferous vegetables against oral pathogens. J Microbiol Biotechnol 26: 2036-2042, 2016.

31. Kim KH, Moon E, Kim SY, Choi SU, Lee JH and Lee KR: 4-Methylthio-butanyl derivatives from the seeds of raphanus sativus and their biological evaluation on anti-inflammatory and antitumor activities. J Ethnopharmacol 151: 503-508, 2014.

32. Yang H, Kang MJ, Hur G, Lee TK, Park IS, Seo SG, Yu JG, Song YS, Park JHY and Lee KW: Sulforaphene suppresses adipocyte differentiation via induction of post-translational degradation of CCAAT/enhancer binding protein beta $(\mathrm{C} / \mathrm{EBP} \beta)$. Nutrients 12: 758, 2020

33. Zhang J, Feng C, Tan X, Hagedoorn PL, Gu C, Xu H and Zhou X Effect of aliphatic diamine spacer length on enzymatic performance of myrosinase immobilized on chitosan microsphere and its application for sulforaphene production. J Biotechnol 299: 79-85, 2019

34. Chen J, Bao C, Kim JT, Cho JS, Qiu S and Lee HJ: Sulforaphene inhibition of adipogenesis via Hedgehog signaling in 3T3-L1 adipocytes. J Agric Food Chem 66: 11926-11934, 2018.

35. Rai R, Gong Essel K, Mangiaracina Benbrook D, Garland J, Daniel Zhao Y and Chandra V: Preclinical efficacy and involvement of AKT, mTOR, and ERK kinases in the mechanism of sulforaphane against endometrial cancer. Cancers (Basel) 12: 1273,2020

36. Biswas R, Ahn JC and Kim JS: Sulforaphene synergistically sensitizes cisplatin via enhanced mitochondrial dysfunction and PI3K/PTEN modulation in ovarian cancer cells. Anticancer Res 35: 3901-3908, 2015.

37. Farooqi AA, de la Roche M, Djamgoz MBA and Siddik ZH: Overview of the oncogenic signaling pathways in colorectal cancer: Mechanistic insights. Semin Cancer Biol 58: 65-79, 2019.

38. Mármol I, Sánchez-de-Diego C, Pradilla Dieste A, Cerrada E and Rodriguez Yoldi MJ: Colorectal carcinoma: A general overview and future perspectives in colorectal cancer. Int J Mol Sci 18: 197, 2017.

39. Chiacchiera F, Matrone A, Ferrari E, Ingravallo G, Lo Sasso G, Murzilli S, Petruzzelli M, Salvatore L, Moschetta A and Simone C: p38alpha blockade inhibits colorectal cancer growth in vivo by inducing a switch from HIF1alpha-to FoxO-dependent transcription. Cell Death Differ 16: 1203-1214, 2009.

40. Li Q, Tang H, Hu F and Qin C: Silencing of FOXO6 inhibits the proliferation, invasion, and glycolysis in colorectal cancer cells. J Cell Biochem 120: 3853-3860, 2019.

41. Krishnamurthy N and KurzrockR: Targeting the Wnt/beta-catenin pathway in cancer: Update on effectors and inhibitors. Cancer Treat Rev 62: 50-60, 2018.

This work is licensed under a Creative Commons Attribution-NonCommercial-NoDerivatives 4.0 International (CC BY-NC-ND 4.0) License. 